\title{
Inhibitory effect of hydroalcoholic and flavonoids extracts of Dracocephalum kotschyi, and its components luteolin, apigenin and apigenin-4'-galactoside on intestinal transit in mice
}

\author{
Hassan Sadraei $^{1^{*}}$, Seyed Mostapha Ghanadian ${ }^{2}$, Saeideh Moazeni ${ }^{1,2}$ \\ ${ }^{1}$ Department of Pharmacology and Toxicology, School of Pharmacy and Pharmaceutical Sciences, Isfahan University of Medical Sciences, Isfahan, I.R. \\ Iran \\ ${ }^{2}$ Department of Pharmacognosy and Isfahan Pharmaceutical Sciences Research Center, School of Pharmacy and Pharmaceutical Sciences, Isfahan \\ University of Medical Sciences, Isfahan, I.R. Iran
}

\section{A R T I C L E IN F O}

Article Type:

Original Article

\section{Article History:}

Received: 1 September 2018

Accepted: 14 November 2018

\section{Keywords:}

Dracocephalum kotschyi

Apigenin

Luteolin

Apigenin-4'-galactoside

Ileum meal transit

\begin{abstract}
A B S T R A C T
Introduction: Dracocephalum kotschyi is an Iranian indigenous herbal plant which has been reported to have antispasmodic activity in vitro. The antispasmodic activity of hydroalcoholic extract of $D$. kotschyi is reported to be due to its flavonoids constituents including apigenin and luteolin. The objective of this research was to compare antispasmodic activities of hydroalcoholic and flavonoids extracts of D. kotschyi on ileum contractions in vivo. In addition, spasmolytic activity of apigenin, luteolin and apigenin-4'-galactoside were compared.

Methods: The hydroalcoholic extract was prepared by percolation method. Flavonoids extract was obtained by solvent-solvent extraction. Antispasmodic effect of the test compounds was assessed by measurement of percent small intestine transit following oral administration of a charcoal meal and compared with control group and standard drug loperamide.

Results: Biochemical assessment of flavonoids content of the extracts showed that ethyl-acetate fraction contained higher quantity of flavonoids. Loperamide $(2.5 \mathrm{mg} / \mathrm{kg})$ reduced charcoal meal movement by $58 \%$ in comparison to control group. Hydroalcoholic extract of $D$. kotschyi $(20 \mathrm{mg} / \mathrm{kg})$ and its ethyl-acetate fraction $(20 \mathrm{mg} / \mathrm{kg})$ reduced the intestinal charcoal meal transit by $32 \%$ and $90 \%$, respectively. Apigenin, luteolin and apigenen-4'-galactoside with oral dose of $20 \mathrm{mg} / \mathrm{kg}$ inhibited intestinal movement of the charcoal meal 93\%, 89\% and $45 \%$, respectively in comparison with the vehicle treated control groups.

Conclusion: This study confirms that both the hydroalcoholic and flavonoids extracts of $D$. kotschyi have antispasmodic properties in vivo. Antimotility of apigenen-4'-galactoside in mice is probably due to release of apigenin in the gastrointestinal tract.
\end{abstract}

Implication for health policy/practice/research/medical education:

This paper provides pharmacological evidence for antimotility of apigenin and luteolin two component of Dracocephalum kotschyi in vivo. In addition it was revealed that inactive apigenin-4'-galactoside in the gastrointestinal tract is converted to active apigenin.

Please cite this paper as: Sadraei H, Ghanadian SM, Moazeni S. Inhibitory effect of hydroalcoholic and flavonoids extracts of Dracocephalum kotschyi, and its components luteolin, apigenin and apigenin-4'-galactoside on intestinal transit in mice. J Herbmed Pharmacol. 2018;9(1):8-13. doi: 10.15171/jhp.2019.02.

\section{Introduction}

Dracocephalum kotschyi Boiss. is a traditional Iranian medicinal plant belonging to Labiatae family (1). D. kotschyi is an aromatic plant enriched in essential oil including carveol, a-pinene, geraniol, a-citral, cyclononadiene, terpinene-4-ol, linalool, neral, myrcene, germacrene-D, isopinocarveol, limonene and a-terpineol (2-4). In recent years several reports have been published on pharmacological activities of D. kotschyi. For instance, the essential oil of D. kotschyi reported to have anti-inflammatory and antinociceptive activities (5). Hydroalcoholic extract of $D$. kotschyi was reported to have antihyperlipidemic effect in an animal model (6) and inhibit tumor proliferation (7-9). Hydroalcoholic extract of $D$. Kotschyi has spasmolytic activities on isolated ileum and uterus $(10,11)$. In addition, D. kotschyi 
extract has also been shown to have immunomodulatory, anti-inflammatory and anti-colitis properties $(12,13)$. It is believed that pharmacological effect of the extract is mainly due to presence of flavonoids constituents (14). Flavonoids are widely distributed in the plant kingdom and occur in many medicinal plants $(13,14)$. The flavonoids constituents of D. kotschyi extract include, apigenin, apigenin 4'-O-beta-D-glucopyranoside, luteolin, luteolin 7-O-beta-D-glucopyranoside, luteolin 3'-O-beta-Dglucuronide, calycopterin, xanthomicrol, isokaempferide, acacetin 7-O-beta-D-glucopyranoside and rosmarinic acid $(15,16)$. It has been reported that calycopterin is responsible for immunomodulatory effect of $D$. kotschyi (17), while apigenin was reported to have antiinflammatory action (18). Among flavonoids constituents of D. kotschyi apigenin and luteolin have potent antispasmodic activities in vitro (19). However, apigenin4'-galacotside shows no significant antispasmodic activity on isolated rat ileum (20). Therefore, it seems that aglycones forms of the flavonoids existed in the plant are pharmacological active constituents. Nevertheless, it is likely that in the gastrointestinal tract glycoside molecule can be removed by gut enzymes and aglycones forms of flavonoids could be released. Therefore, first objective of this research was to investigate the antimotility effect of D. kotschyi flavonoids rich fraction with hydroalcoholic extract in vivo. The second objective was to compare antimotility of luteolin and apigenin with that of apigenin4 '-galactose as well as the total extracts to find out which have stronger antimotility activities.

\section{Materials and Methods}

Dracocephalum kotschyi aerial parts were collected from a cultivated farm in Fereydun-Shahr mountain base (in Isfahan province, Iran) and identified at the Botany Department of the Faculty of Sciences, University of Isfahan. A voucher specimen was deposited at the herbarium of the School of Pharmacy and Pharmaceutical Sciences of Isfahan University of Medical Sciences (No. 1519).

The plant materials were dried in shade and ground to powder using electrical miller (Keep, Korea). The powdered materials were subjected to extraction by $70 \%$ ethanol in a percolator apparatus. The ratio of plant powder to solvent for hydroalcoholic extract was 1:8. The solvent was evaporated under vacuum using rotary (Heidolph, Germany) at $70^{\circ} \mathrm{C}$ and the yield of hydroalcoholic extract was determined. The hydroalcoholic extract (100 g) then was subjected to solvent-solvent extraction using chloroform/water mixture (1:1) and shacked thoroughly and the chloroform layer was decanted. Fresh chloroform was added and the process was repeated twice. The aqueous layer was then mixed with ethyl-acetate $(2: 1)$ and shacked as before. The ethyl-acetate layer was decanted and the fresh ethyl-acetate was added and the process was repeated five times. These fractions were concentrated under vacuum using rotary at $30^{\circ} \mathrm{C}$ for ethyl-acetate and at $90^{\circ} \mathrm{C}$ for the aqueous fraction (21-23). The yield of dried extract was calculated for each fraction.

Biochemical standardization of the extract

Total flavonoids content of the hydroalcoholic extract and other fractions were determined by aluminum chloride colorimetric method (24). Fifty milligrams of quercetin was dissolved in $50 \mathrm{~mL}$ methanol and then diluted to 4 , 20, 100 and $500 \mu \mathrm{g} / \mathrm{mL}$. The diluted standard solutions $(0.1 \mathrm{~mL})$ were separately mixed with $0.1 \mathrm{~mL}$ of $20 \%$ aluminum chloride, $0.1 \mathrm{~mL}$ of glacial acetic acid and 2.7 $\mathrm{mL}$ of methanol. After incubation at room temperature for 40 minutes in the dark, the absorbance of the reaction mixture was measured at $415 \mathrm{~nm}$ with a Jenway $5105 \mathrm{U} . \mathrm{V} / \mathrm{V}$ spectrophotometer (England). The spectrophotometer was initially calibrated with blank solution. In the blank solution, aluminum chloride was substituted by the same amount of distilled water. The assessment was repeated 3 times. Similarly, $0.1 \mathrm{~mL}$ of hydroalcoholic extract or other fraction solutions were reacted with aluminum chloride for determination of their total flavonoids content.

\section{Drugs and solutions}

The following drugs and solutions were used in this research: D. kotschyi hydroalcoholic extract, aqueous and ethyl-acetate fractions, loperamide, apigenin, luteolin and apigenin-4'-galacotside. The hydroalcoholic extract was made up as 10 and $40 \mathrm{mg} / \mathrm{mL}$ stock solution in 50\% ethanol and diluted in distilled water to obtain concentrations of 1 , 4 and $10 \mathrm{mg} / \mathrm{mL}$. Aqueous and ethyl-acetate fractions were made up as 10 and $50 \mathrm{mg} / \mathrm{mL}$ stock solution in ethanol and further serial dilution was made in distilled water ( 1 and $5 \mathrm{mg} / \mathrm{mL}$ ). Loperamide was dissolved in ethanol as $1 \mathrm{mg} /$ $\mathrm{mL}$ stock solution and was further diluted with distilled water $(100 \mu \mathrm{g} / \mathrm{mL})$. Apigenin, luteolin (Aktin Chemical Ins. China) and apigenin-4'-galacotside was made up as 4 and $20 \mathrm{mg} / \mathrm{mL}$ stock suspension or solution in DMSO. Further dilution was made in distilled water $(0.2$ and $1 \mathrm{mg} / \mathrm{mL}$ in 5\% DMSO, respectively). Charchol (3\%) plus tragacanth powder (5\%) suspension was prepared in distilled water. Unless stated, all the chemicals were purchased from Merck (Germany).

\section{Pharmacological studies}

Male albino mice (20-30 g), bred in School of Pharmacy and Pharmaceutical Sciences (Isfahan University of Medical Sciences, Iran) animal house, were kept at room temperature. The animals were fasted overnight prior to the experiments with free access to water. All animals were handled in accordance with the internationally accepted principles for laboratory animal use and care (25). In this study effect of hydroalcoholic and aqueous and ethyl-acetate fractions, loperamide $(2.5 \mathrm{mg} / \mathrm{kg})$, apigenin 
and luteolin $(2.5,5,10$ and $20 \mathrm{mg} / \mathrm{kg})$ and apigenin-4'galacotside $(5,10,20$ and $40 \mathrm{mg} / \mathrm{kg})$ were examined on gut motility using charcoal meal transit test. All experiments were conducted in parallel with control groups treated with equivalent volume of the vehicle. Each dose of drug was examined on 10 separate mice. Stock solution was adjusted in such way that each mouse was given $0.5 \mathrm{~mL}$ of test drugs or extracts.

\section{Charcoal meal transit test}

In this experiment, movement of charcoal meal in the small intestine was assessed. For this purpose D. kotschyi hydroalcoholic extract (5, 20, 80 and $120 \mathrm{mg} / \mathrm{kg}$ ), flavonoids extract ( 5 and $20 \mathrm{mg} / \mathrm{kg}$ ), apigenin and luteolin $(2.5,5,10$ and $20 \mathrm{mg} / \mathrm{kg})$ and apigenin-4'-galacotside (5, 10, 20 and $40 \mathrm{mg} / \mathrm{kg}$ ) or loperamide $(2.5 \mathrm{mg} / \mathrm{kg})$ was given orally to mice and 45 minutes later $0.5 \mathrm{~mL}$ of charcoal meal containing 3\% charcoal plus 5\% tragacanth suspension was administered orally. Thirty minutes after charcoal meal administration, each animal was sacrificed and distance of charcoal movement in the small intestine was measured.

Measurement and statistical analysis

Ileum transit was expressed as percentage of charcoal moved from pylorus to the caecum relative to the whole length of the ileum. All results were expressed as mean \pm standard error of mean (SEM) and compared with corresponding vehicle-treated control group using unpaired Student's $t$ test. $P$ values less than 0.05 was considered statistically significant. SigmaPlot computer program (version 11) was used for statistical analysis and plotting the graphs.

\section{Results}

The yield of hydroalcoholic extract was $23 \%$ (W/W). Amounts of total flavonoids content were $0.36 \%, 0.003 \%$, $0.1 \%$ and $1.12 \%$ for hydroalcoholic, chloroform, aqueous and ethyl-acetate fractions, respectively.

\section{Charcoal meal transit test}

In the control group treated with distilled water, charcoal meal moved up to $97 \%$ of small intestine within 30 minutes of oral administration of charcoal meal. Loperamide $(2.5 \mathrm{mg} / \mathrm{kg})$, tested as a standard drug, reduced charcoal movement by $58 \%$ relative to the vehicle treated control group. There was no statistically significant difference between distilled water treated control and the control group treated with vehicle (ethanol) (Figure 1). Hydroalcoholic extract of D. kotschyi with dose of $5 \mathrm{mg} / \mathrm{kg}$ had no significant inhibitory effect on intestinal charcoal meal transit but with dose of $20 \mathrm{mg} /$ $\mathrm{kg}$ significantly reduced meal transit by $32 \%$. When the dose of hydroalcoholic extract was increased to $80 \mathrm{mg} /$ $\mathrm{kg}$ intestinal transit was inhibited by $70 \%$ compared to vehicle treated control group (Figure 2). Further increase in dose of $D$. kotschyi hydroalcoholic extract $(120 \mathrm{mg} / \mathrm{kg})$ had no more inhibitory effect on intestinal meal transit.

Ethyl-acetate fraction prepared from $D$. kotschyi extract had more pronounced inhibitory effect on charcoal meal transit compared to hydroalcoholic extract. Oral dose of $5 \mathrm{mg} / \mathrm{kg}$ ethyl-acetate fraction reduced intestinal transit by $45 \%$ relative to vehicle treated control group. Ethylacetate fraction of D. kotschyi with oral dose of $20 \mathrm{mg} /$ $\mathrm{kg}$ inhibited the charcoal meal transit by $90 \%$ (Figure 1).

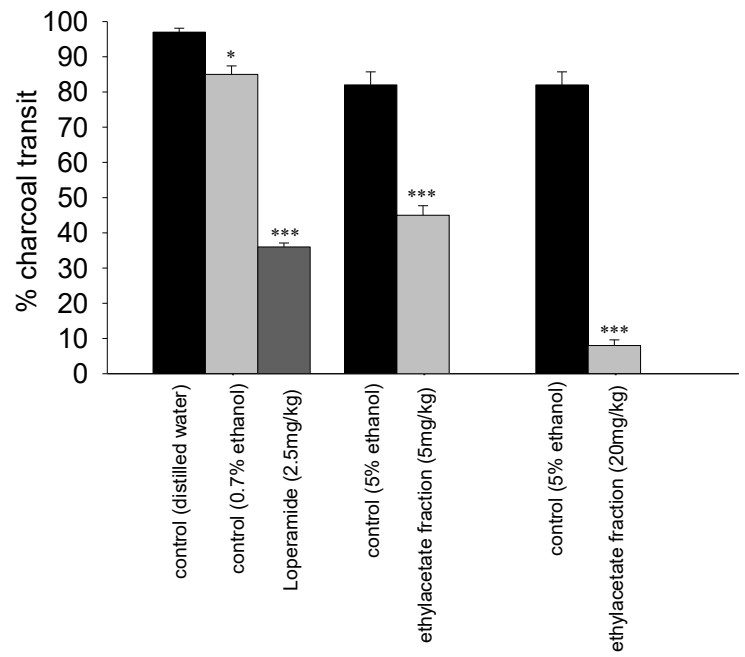

Figure 1. Effect of loperamide and ethyl-acetate fraction of $D$. kotschyi on intestinal distance moved by charcoal meal $(0.5 \mathrm{~mL}$; per orum) in comparison with the vehicle treated control group in mice. Data are expressed as mean \pm SEM $(n=10)$. Stars show degree of statistically significant difference in comparison with its appropriate control group $\left({ }^{*} P<0.05,{ }^{* *} P<0.001\right.$, Student's $t$ test).

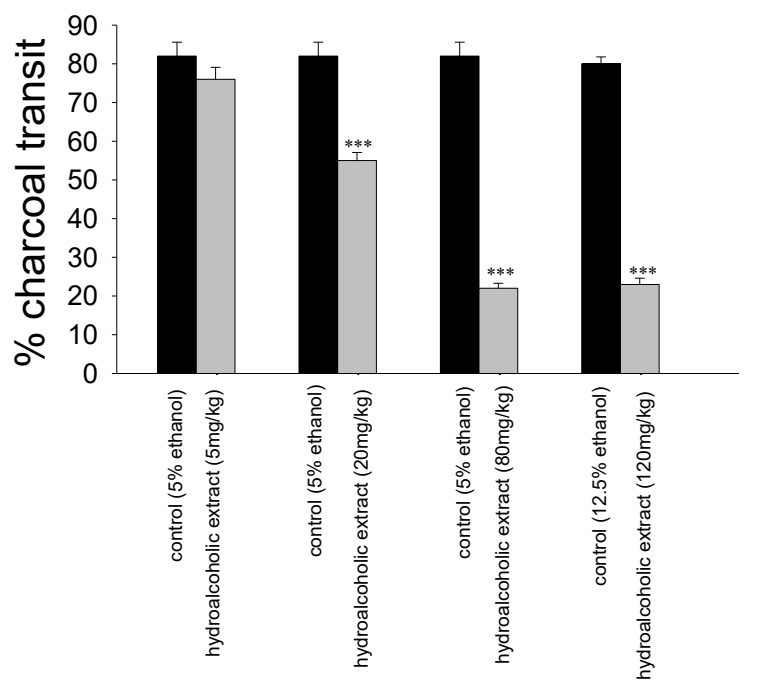

Figure 2. Effect of hydroalcoholic extract of $D$. kotschyi on intestinal distance moved by charcoal meal $(0.5 \mathrm{~mL}$; per orum) in comparison with the vehicle treated control group in mice. Data are expressed as mean \pm SEM $(n=10)$. Stars show degree of statistically significant difference in comparison with its appropriate control group $\left({ }^{* * *} \mathrm{P}<0.001\right.$, Student's $t$ test). 
Remaining aqueous fraction up to dose of $120 \mathrm{mg} / \mathrm{kg}$ exhibited no inhibitory effect on intestinal charcoal meal transit (Figure 3).

Luteolin and apigenin are two known flavonoids which have been identified in $D$. kotschyi extract. Both these flavonoids components in a dose dependent manner inhibited mice intestinal motility. Luteolin and apigenin at oral dose of $2.5 \mathrm{mg} / \mathrm{kg}$ reduced intestinal charcoal meal transit by $40 \%$ and $42 \%$, respectively compared to vehicle treated control group (Figures $4 \& 5$ ). With oral dose of $20 \mathrm{mg} / \mathrm{kg}$, movement of transit meal was inhibited by $89 \%$ and $93 \%$, respectively. Apigenin-4'-galacotside with oral dose of $5 \mathrm{mg} / \mathrm{kg}$ had no significant effect on charcoal meal transit. However, with higher doses (10, 20 and $40 \mathrm{mg} / \mathrm{kg}$ ) significantly inhibited intestinal transit by $38 \%, 45 \%$ and

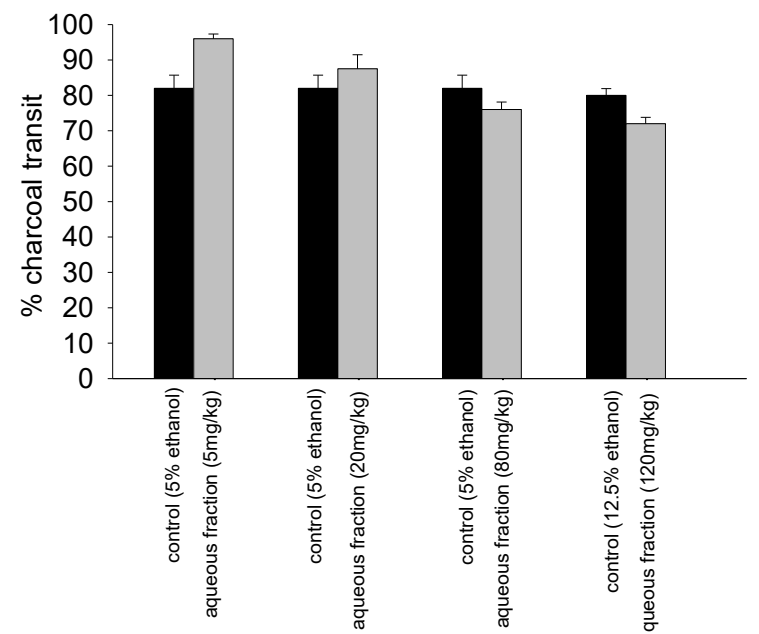

Figure 3. Effect of aqueous fraction of $D$. kotschyi on intestinal distance moved by charcoal meal $(0.5 \mathrm{~mL}$; per orum) in comparison with the vehicle treated control group in mice. Data are expressed as mean \pm SEM $(n=10)$. There is no statistically significant difference between test and control groups.

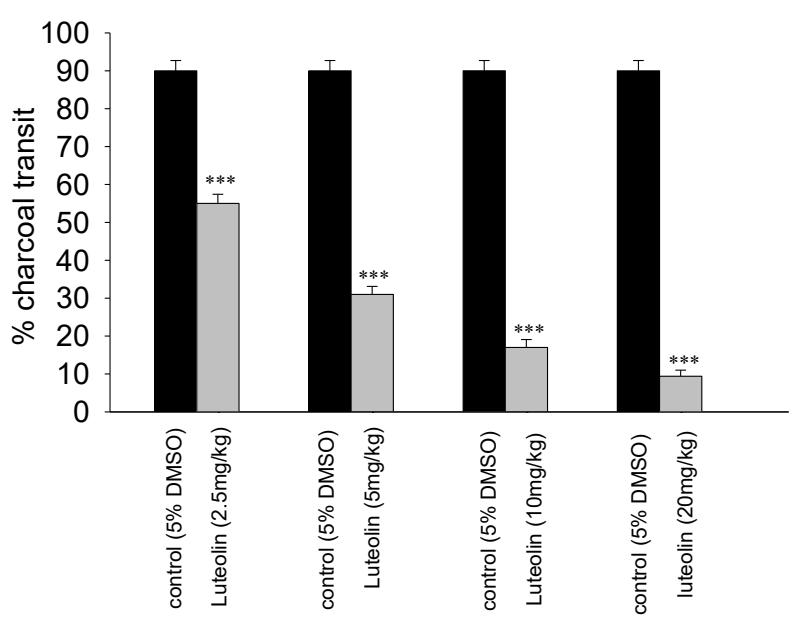

Figure 4. Effect of luteolin on intestinal distance moved by charcoal meal $(0.5 \mathrm{~mL}$; per orum) in comparison with the vehicle treated control group in mice. Data are expressed as mean \pm SEM $(n=10)$. Stars show degree of statistically significant difference in comparison with its appropriate control group ( ${ }^{* *} P<0.001$, Student's $t$ test).
$55 \%$, respectively (Figure 6). In the control groups treated with vehicle (DMSO) there were no statistically significant differences compared with distilled water treated group.

\section{Discussion}

Hydroalcoholic extract of D. kotschyi possessed spasmolytic activity on isolated rat ileum and uterus $(10,11)$. In addition, D. kotschyi extract has been shown to reduce diarrhea induced in mice (26). Furthermore, it has been shown to have anti-colitis activity in animal model of colitis (12). Antispasmodic activity of D. kotschyi extract has been attributed to its flavonoids contents including luteolin and apigenin (20). In this research we have focused on antispasmodic action of hydroalcoholic and flavonoids extracts of D. kotschyi in whole animal to

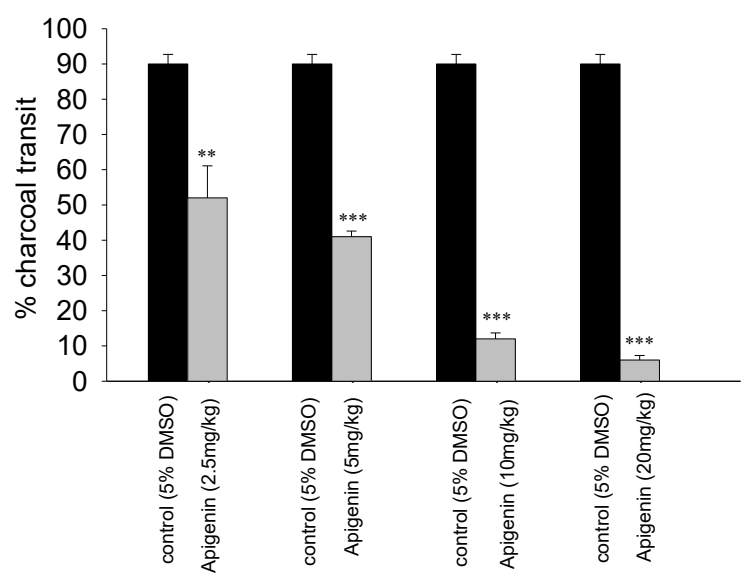

Figure 5. Effect of apigenin on intestinal distance moved by charcoal meal $(0.5 \mathrm{~mL}$; per orum $)$ in comparison with the vehicle treated control group in mice. Data are expressed as mean \pm SEM $(n=10)$. Stars show degree of statistically significant difference in comparison with its appropriate control group $\left({ }^{* *} P<0.05,{ }^{* * *} P<0.001\right.$, Student's $t$ test).

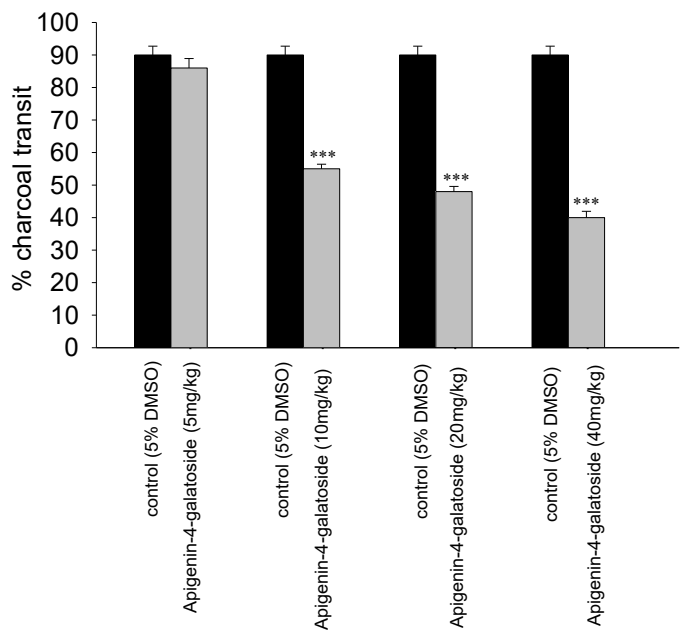

Figure 6. Effect of apigenin-4'-galactoside on intestinal distance moved by charcoal meal $(0.5 \mathrm{ml}$; per orum $)$ in comparison with the vehicle treated control group in mice. Data are expressed as mean \pm SEM $(n=10)$. Stars show degree of statistically significant difference in comparison with its appropriate control group $\left({ }^{\star * *} P<0.001\right.$, Student's $t$ test) 
find out which one would be more suitable remedy for gastrointestinal aliments. Furthermore, antispasmodic actions of three components of $D$. kotschyi extract were also assessed in vivo.

Hydroalcoholic extract contains both polar and nonpolar constituents while the chloroform fraction mainly contains non-polar constituents including non-active substances such as chlorophyll. In the mixture of water and ethyl-acetate, polar substances would be dissolves in the aqueous part while less polar substances including flavonoids are dissolved in ethyl-acetate layer. Biochemical assay also confirm that ethyl-acetate fraction is enrich in flavonoids. Inhibition of small intestine transit also confirms that more active substances are concentrated in the flavonoids rich fraction. Measurement of transit of meal in the small intestine has been used for assessment of gut motility in intact animal. It has been shown that hydroalcoholic extract of $D$. kotschyi with oral doses of 20 and $80 \mathrm{mg} / \mathrm{kg}$ caused significant inhibition of intestinal motility. Furthermore, increase in dose had no further inhibitory effect indicating that it is possible that other components presence in the extract cause its inhibitory action. As mentioned above most of flavonoids components are concentrated in the ethyl-acetate fraction. Ethyl-acetate fraction with dose of $20 \mathrm{mg} / \mathrm{kg}$ reduced intestinal motility by $90 \%$ while hydroalcoholic extract reduced by $32 \%$. This indicates that flavonoids extract is pharmacologically more active than the hydroalcoholic extract. Luteolin and apigenin are two flavonoids component of D. kotschyi with antimotility activities. Therefore, it can be concluded that they have major contribution in the antispasmodic activity of $D$. kotschyi extract. Comparison of antimotility of luteolin and apigenin with similar doses indicates that they have similar pharmacological activities on intestinal meal transit. On the other hand, apigenin-4'-galacotside was less active in comparison to apigenin. However, unlike rat isolated ileum (20), apigenin-4'-galacotside inhibited gut motility in vivo which is a good indication that in the gastrointestinal tract, the galactoside molecule is removed and aglycone apigenin is released. Therefore, it is very likely that the observed inhibitory effect of apigenin-4'galacotside is due to action of apigenin.

\section{Conclusion}

In conclusion this study clearly shows the antimotility effect of $D$. kotschyi extract in intact animal and indicates that antispasmodic effect of extract is mainly due to its flavonoids contents including apigenin and luteolin. As galactoside form of apigenin and luteolin lack antispasmodic activity in vitro, this study indicates that in the gastrointestinal tract, the sugar moiety is removed and active aglycones flavonoids are released.

\section{Acknowledgments}

This project was part of Pharm. D thesis of Saeideh
Moazeni as Pharm. D student at Isfahan University of Medical Sciences.

\section{Authors' contributions}

MG was responsible for preparation of extract while HS supervised the pharmacological studies. SM was responsible for performing the laboratory works. All contributed to preparation of the article and confirmed final edition for publication.

\section{Conflict of interests}

None.

\section{Ethical considerations}

Ethical issues have been approved by Isfahan University of Medocal Sciences ethical committee (No. 397538).

\section{Funding/Support}

This research project financially was supported by Research Department of Isfahan University of Medical Sciences (Project No. 397538).

\section{References}

1. Naghibi F, Mosaddegh M, Mohammadi Motamed M, Ghorbani A. Labiatae family in folk medicine in Iran: from ethnobotany to pharmacology. Iranian J Pharm Res. 2010;4(2):63-79.

2. Yaghmai M, Taffazoli R. The essential oil of Dracocephalum kotschyi Boiss. Flavour Fragrance J. 1988;3:33-36.

3. Saeidnia S, Gohari AR, Uchiyama N, Ito M, Honda G, Kiuchi F. Two new monoterpene glycosides and trypanocidal terpenoids from Dracocephalum kotschyi. Chem pharm bull. 2004;52(10):1249-50.

4. Sadraei H, Asghari G, Kasiri F. Comparison of antispasmodic effects of Dracocephalum kotschyi essential oil, limonene and alpha-terpineol. Res pharm Sci. 2015;10(2):109-16.

5. Golshani S, Karamkhani F, Monsef-Esfehani HR, Abdollahi M. Antinociceptive effects of the essential oil of Dracocephalum kotschyi in the mouse writhing test. J Pharm Pharm Sci. 2004;7(1):76-9.

6. Sajjadi ES, Movahedian Atar A, Yektaian A. Antihyperlipidemic effect of hydroalcoholic extract, and polyphenolic fraction from Dracocephalum kotschyi Boiss. Pharm Acta Helv. 1998;73(3):167-70.

7. Talari M, Seydi E, Salimi A, Mohsenifar Z, Kamalinejad M, Pourahmad J. Dracocephalum: novel anticancer plant acting on liver cancer cell mitochondria. BioMed Res Int. 2014; 40(5): 1-10. doi: 10.1155/2014/892170.

8. Moghaddam G, Ebrahimi SA, Rahbar-Roshandel N, Foroumadi A. Antiproliferative activity of flavonoids: influence of the sequential methoxylation state of the flavonoid structure. Phytother Res. 2012;26:1023-28. 
doi: $10.1002 /$ ptr.3678.

9. Jahaniani F, Ebrahimi SA, Rahbar-Roshandel N, Mahmoudian M. Xanthomicrol is the main cytotoxic component of Dracocephalum kotschyi and a potential anti-cancer agent. Phytochemistry. 2005;66:1581-92. doi:10.1016/j.phytochem.2005.04.035.

10. Sadraei H, Asghari G, Kasiri F. Antispasmodic effect of Dracocephalum kotschyi hydroalcoholic extract on rat ileum contraction. Res Pharm Sci. 2015;10(5):44652.

11. Sadraei H, Asghari G, Alinejad M. Comparison of antispasmodic effect of hydroalcoholic extract of Dracocephalum kotschyi Boiss. in rat uterus and ileum. Res Pharm Sci. 2016;11(4):284-92. doi: 10.4103/1735-5362.189295.

12. Sadraei H, Asghari G, Khanabadi M, Minaiyan M. Anti-inflammatory effect of apigenin and hydroalcoholic extract of Dracocephalum kotschyi on acetic acid-induced colitis in rats. Res Pharm Sci. 2017;12(4):322-9. doi: 10.4103/1735-5362.212050.

13. Kilani-Jaziri S, Mustapha N, Mokdad-Bzeouich I, EI Gueder D, Ghedira K, Ghedira-Chekir L. Flavones induce immunomodulatory and anti-inflammatory effects by activating cellular anti-oxidant activity: a structure-activity relationship study. Tumor Biol. 2016;37:6571-79. doi: 10.1007/s13277-015-4541-5.

14. Havsteen B. Flavonoids, a class of natural products of high pharmacological potency. Biochem Pharmacol. 1983;32:1141-8.

15. Gohari AR, Saeidnia S, Matsuo K, Uchiyama N, Yagura T, Ito M, Kiuchi F, Honda G. Flavonoid constituents of Dracocephalum kotschyi growing in Iran and their trypanocidal activity. Nat Med. 2003;57(6):250-2.

16. Fattahi M, Nazeri V, Torras-Claveria L, Sefidkon F, Cusido RM, Zamani Z, et al. Identification and quantification of leaf surface flavonoids in wildgrowing populations of Dracocephalum kotschyi by LC-DAD-ESI-MS. Food chem. 2013;141(1):139-46. doi: $\quad$ 10.1016/j.foodchem.2013.03.019.

17. Faham N, Javidnia K, Bahmani M, Amirghofran Z. Calycopterin, an immunoinhibitory compound from the extract of Dracocephalum kotschyi. Phytother Res. 2008;22(9):1154-8. doi: 10.1002/ptr.2382.

18. Lee JH, Zhou HY, Cho SY, Kim YS, Lee YS Jeong CS. Anti-inflammatory mechanism of apigenin: inhibition of cyclooxygenase expression, adhesion of monocytes to human unbilical vein endothelial cells and expression of cellular adhesion molecules. Arch Pharm Res. 2007;30(10):1318-27.

19. Sadraei H, Ghanadian M, Asghari G, Sekhavati N. Antispasmodic activity of apigenin and luteolin, two components of Dracocephalum kotschyi extract, on rat ileum contractions. J Herbmed Pharmacol. 2018;7(2):100-5. doi: 10.15171/jhp.2018.17

20. Sadraei H, Ghanadian M, Asghari G, Sekhavati N. Bioactivity guided isolation of active fraction of Dracocephalum kotschyi extract responsible for antispasmodic activity on rat ileum. [thesis]. Isfahan, Iran: School of Pharmacy, Isfahan University of Medical Sciences and Health Services; 2018.

21. Samuelsson G. Drugs of Natural Origin. Stockholm: Swedish Pharmaceutical Press: Sweden; 1999:48-9.

22. Ghasemi Dehkordi NA, Sajadi SE. Iranian Herbal Pharmacopoeia. Tehran: Ministry of Health Pub. 2002.

23. Harborn JB, Mabary TJ, Mabary H. Physiological and functional of flavonoids. Phytochemical Methods. Flavonoids. 1975:970-1055.

24. Chang CC, Yang MH, Wen HM, Chern JC. Estimation of Tatal Flavonoid Content in Propolis by Two Complementary Colorimetric Methods. J Food Drug Anal. 2002;10(3): 178-82.

25. Committee for the update of the guide for the care and use of laboratory animals National Research Council. Guide for the Care and use of Laboratory animals. Washington DC: The National Academies Press; 2010. p. 11-37.

26. Sadraei H, Asghari G, Shahverdi F. Antidiarrhoeal assessment of hydroalcoholic and hexane extracts of Dracocephalum kotschyi Boiss. and apigenin in mice. Res Pharm Sci. 2016;11(3):200-9. 